\title{
Ethylenediaminetetraacetic Acid Chelation for Band Keratopathy before Ab Interno Glaucoma Surgery
}

\author{
Tomoki Shirakami*, Yasuyuki Takai, Mihoko Mochiji, Masaki Tanito \\ Department of Ophthalmology, Faculty of Medicine, Shimane University, Shimane, Japan \\ Email: ^t-shira@med.shimane-u.ac.jp
}

How to cite this paper: Shirakami, T., Takai, Y., Mochiji, M. and Tanito, M. (2019) Ethylenediaminetetraacetic Acid Chelation for Band Keratopathy before $\mathrm{Ab}$ Interno Glaucoma Surgery. Open Journal of Ophthalmology, 9, 165-167.

https://doi.org/10.4236/ojoph.2019.94018

Received: September 2, 2019

Accepted: October 8, 2019

Published: October 11, 2019

Copyright $\odot 2019$ by author(s) and Scientific Research Publishing Inc. This work is licensed under the Creative Commons Attribution International License (CC BY 4.0). http://creativecommons.org/licenses/by/4.0/

\section{Open Access}

\begin{abstract}
An 87-year-old woman with primary open-angle glaucoma presented to our hospital. Although the combined cataract and minimally invasive glaucoma surgeries (MIGS) were an appropriate surgical option, the presence of band keratopathy made it difficult to perform ab interno glaucoma surgery in her right eye (OD); therefore, the corneal opacity was removed using ethylenediaminetetraacetic acid (EDTA) chelation procedure. One month after chelation, microhook ab interno trabeculotomy and cataract surgery were performed successfully. Clear intraoperative visualization of the angle structures is critical for the success of these MIGS procedures. In glaucomatous eyes that require MIGS, EDTA chelation is a good neoadjuvant therapy for coexisting band keratopathy.
\end{abstract}

\section{Keywords}

Microhook Ab Interno Trabeculotomy, Minimally Invasive Glaucoma Surgery (MIGS), Band Keratopathy, Primary Open-Angle Glaucoma, Ethylene-Diamine-Tetra-Acetic Acid (EDTA) Chelation

\section{Introduction}

$\mathrm{Ab}$ interno approach is used in new minimally invasive glaucoma surgery (MIGS) procedures including the Trabectome (NeoMedix Corporation, Tustin, CA), Kahook Dual Blade (New World Medical, Rancho Cucamonga, CA), microhook ab interno trabeculotomy, and 360-degree suture trabeculotomy, instead of the ab externo approach as in conventional trabeculotomy [1]. However, clear intraoperative visualization of the angle structures is critical for the success of these MIGS procedures. Here we report a case with concurrent glaucoma and 
band keratopathy that removed corneal opacity prior to glaucoma surgery to gain the visibility of angle structure during the MIGS.

\section{Case}

An 87-year-old woman with primary open-angle glaucoma presented to our hospital for poor drug adherence and progression of damage to the visual field in her right eye (OD). At referral, the best-corrected visual acuity (BCVA) and intraocular pressure (IOP) were 0.8 and $17 \mathrm{mmHg}$, respectively, with use of four antiglaucoma medications OD. Although the combined cataract and minimally invasive glaucoma surgeries (MIGS) was an appropriate surgical option, the presence of band keratopathy (Figure 1(a)) made it difficult to perform ab interno glaucoma surgery OD; therefore, the corneal opacity was removed using ethylenediaminetetraacetic acid (EDTA) chelation procedure. The procedure involved corneal epithelium removal and subsequent debridement with a sponge (M.Q.A., Inami, Tokyo, Japan) soaked with $0.05 \mathrm{~mol} / \mathrm{l}$ EDTA [2]. One month after chelation, microhook ab interno trabeculotomy and cataract surgery [3] [4] were performed (Figure 1(b)). Before trabeculotomy, phacoemulsification/aspiration and intraocular lens implantation were performed through a 2.4-mm-wide clear sclerocorneal incision. Under visualization using a Swan-Jacob gonioprism lens (Ocular Instruments, Bellevue, WA), the inner wall of Schlemm's canal and trabecular meshwork were incised over 3 clock hours nasally (Figure 1(c)) and temporally (Figure 1(d)) using Tanito ab interno trabeculotomy microhooks (Inami) inserted through the corneal side ports. No complications other than early postsurgical hyphema developed perioperatively. At the final visit 8 months

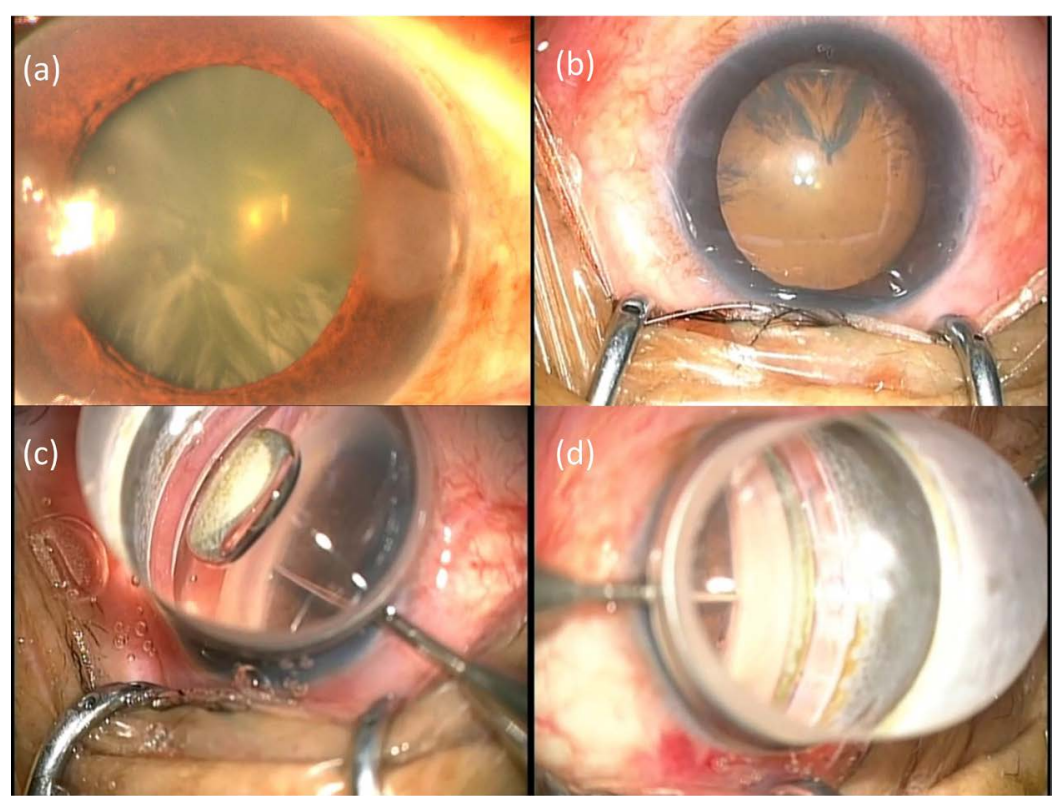

Figure 1. Perioperative findings. (a) At referral, band keratopathy and a cataract are observed in the right eye. (b) One month after EDTA chelation, no dense corneal opacity is observed. Under the Swan-Jacob gonioprism, the angle structures are seen clearly during the microhook ab interno trabeculotomy (c) nasally and (d) temporally. 
postoperatively, the BCVA and IOP were 0.7 and $10 \mathrm{mmHg}$, respectively, with use of three antiglaucoma medications OD.

\section{Discussion}

Clear intraoperative visualization of the angle structures is critical for the success of these MIGS procedures. There are no reports that cases with corneal opacity are underwent MIGS. In this case, clear visualization of the angle structures was acquired by EDTA chelation. Chelation of calcium with topical EDTA is a safe and reliable method for removing band keratopathy with a low retreatment rate [2].

\section{Conclusion}

We report a case with concurrent glaucoma and band keratopathy that removed corneal opacity prior to glaucoma surgery to gain the visibility of angle structure during the MIGS. In glaucomatous eyes that require MIGS, EDTA chelation is a good neoadjuvant therapy for coexisting band keratopathy.

\section{Consent}

The authors have received written informed consent from the patient.

\section{Conflicts of Interest}

The microhooks used were co-developed by Masaki Tanito, $\mathrm{MD}, \mathrm{PhD}$, and $\mathrm{In}$ ami \& Co., Ltd. (Tokyo, Japan). Dr. Tanito receives royalties from Inami \& Co., Ltd.

\section{References}

[1] Tanito, M. and Matsuo, M. (2019) Ab-Iunterno Trabeculotomy-Related Glaucoma Surgeries. Taiwan Journal of Ophthalmology, 9, 67-71. https://doi.org/10.4103/tjo.tjo $38 \quad 19$

[2] Al-Hity, A., Ramaesh, K. and Lockington, D. (2018) EDTA Chelation for Symptomatic Band Keratopathy: Results and Recurrence. Eye, 32, 26-31. https://doi.org/10.1038/eye.2017.264

[3] Tanito, M., Sano, I., Ikeda, Y. and Fujihara, E. (2017) Short-Term Results of Microhook Ab Interno Trabeculotomy, A Novel Minimally Invasive Glaucoma Surgery in Japanese Eyes: Initial Case Series. Acta Ophthalmologica, 95, e354-360. https://doi.org/10.1111/aos.13288

[4] Tanito, M., Ikeda, Y. and Fujihara, E. (2017): Effectiveness and Safety of Combined Cataract Surgery and Microhook Ab Interno Trabeculotomy in Japanese Eyes with Glaucoma: Report of an Initial Case Series. Japanese Journal of Ophthalmology, 61 , 457-464. https://doi.org/10.1007/s10384-017-0531-z 